\title{
Improved Intelligent Vehicle Localization Using Magnetic Ruler
}

\author{
Chunxiang WANG \\ Research Institute of Robotics, Shanghai Jiao Tong University \\ Shanghai, 200240, China \\ Ming YANG*, Bing WANG \\ Department of Automation, Shanghai Jiao Tong University \\ Key Laboratory of System Control and Information Processing, Ministry of Education of China \\ Shanghai, 200240, China \\ Haigui XU \\ Research Institute of Robotics, Shanghai Jiao Tong University \\ Shanghai, 200240, China \\ Hao LI \\ The Robotics Laboratory, Mines Paris (ParisTech) / INRIA-IMARA Team \\ Le Chesnay, B.P.105,78153 France \\ Received: 10-03-2011 \\ Accepted: 19-04-2011
}

\begin{abstract}
This paper proposes an improved intelligent vehicle localization method, which integrates the data from the odometry and the magnetic ruler(MR). The MR is used to detect the magnetic markers, which provides the absolute positioning reference. The data from odometry and the MR are used to localize the vehicle by using unscented Kalman filter. The data association (DA) method is adopted to validate the observations from the MR. The experiment results show that the method is a robust way.
\end{abstract}

Keywords: Unscented Kalman filter, Dead reckoning, Data association, Magnetic ruler, Intelligent vehicle localization

\section{Introduction}

Dead reckoning is a fundamental position estimation method, which is most widely used in the intelligent vehicle localization. However, intelligent vehicle cannot only rely on dead reckoning to determine their locations owing to the accumulative errors. The vehicle must be equipped with external sensors that obtain information from the environment to help the vehicle determine its location more accurately. Most researchers utilized GPS, ultrasonic $^{1-3}$, optical flow sensor or machine vision based systems to provide the positioning information. The precision or reliability of results can be improved with several sensors ${ }^{4-7}$. However, the problems associated with using GPS for vehicle navigation are insufficient position accuracy and signal blockage due to buildings and hilly terrain. The sensing system based on an optical technique such as machine vision is easy susceptible to the influence of shade, snow, fog or other environmental conditions.

*Corresponding author: mingyang@sjtu.edu.cn 
Position measurement using magnetic markers has been proved to be one promising technology for intelligent vehicle guidance and control ${ }^{8-9}$. The California Partners for Advanced Transit and Highways (PATH) program has deployed the magnetic marker system in conjunction with its experimental vehicles in a number of international and national demonstrations ${ }^{10}$. L. Conde Bento $^{11}$, utilized magnetic markers to provide the absolute positioning reference for autonomous vehicles navigation in semi-structured outdoor environments. No physical contact is needed to produce measures. The magnetic marker reliability is independent of weather conditions or environmental conditions, and maintenance requirement is low since the system utilizes passive rather than active markers.

To implement the functions of the integrated system and improve the location precision and the reliability of the vehicle localization system, it is necessary to adopt some sensor fusion algorithms, which can estimate and eliminate some sensor errors (such as wheel slippage or roughness of the ground). The best known algorithm to solve the problem of nonlinear filtering is extended Kalman filter (EKF) ${ }^{12}$. The series approximations in the EKF algorithm can, however, lead to poor representations of the nonlinear functions and probability distributions of interest.

The approach to implement sensor fusion for a highly nonlinear system is unscented Kalman filter (UKF), which can calculate the posterior covariance accurately to the $3^{\text {rd }}$ order Taylor series expansion of the nonlinear state functions and measurement functions ${ }^{13}$. The UKF was used to fuse the data from the odometry with the data from the magnetic ruler and showed better results than the $\mathrm{EKF}^{14-15}$. However, in the localization system based on the magnetic marker, while the lateral offset from the magnetic ruler suffers from large disturbance or the magnetic ruler detects the fault marker, the large error will occur in position estimation. L. C. Bento adopted the data association method in the EKF framework for accepting/rejecting observations from the magnetic sensing system ${ }^{11}$.

This paper integrates the data from the odometry and the magnetic ruler by using the UKF. An improved intelligent vehicle localization method is realized by adopting the data association method to judge the validity of the observations from the magnetic ruler. The localization of the intelligent vehicle based on the odometry and the magnetic ruler is presented in
Sections 2.1 and 2.2. In Section 3, the sensor fusion process based on UKF is described in detail and the improved sensor fusion algorithm is realized by introducing the data association method in the UKF. The experimental results and conclusions are given in Section 4 and 5, respectively.

\section{Localization System}

Two different types of sensors are utilized in the intelligent vehicle localization system. Odometry is used for dead reckoning of the vehicle. Owing to the accumulative errors in the dead reckoning process, poor position estimation may occur. The magnetic ruler in the magnetic sensing system is used to detect the magnetic markers embedded under the road surface and provide the absolute positioning reference. Therefore, in order to accurately estimate the position of the vehicle, it is necessary to fuse the data from the two sensing systems.

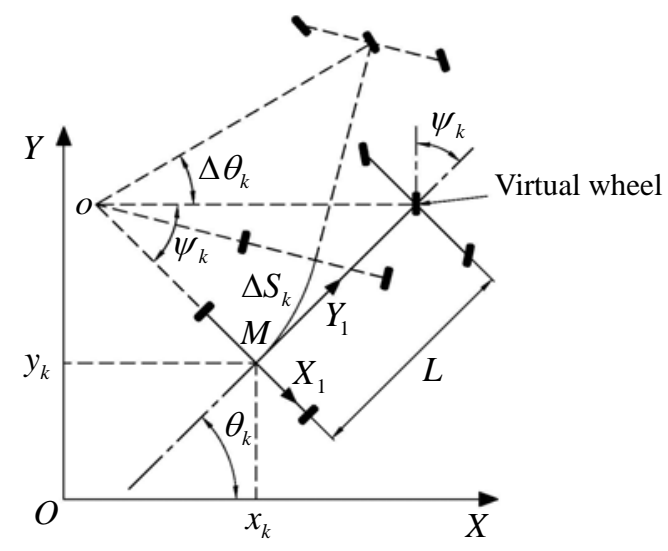

Fig. 1. Vehicle Kinematics

\subsection{Odometry}

The intelligent vehicle is equipped with one driving encoder and one steering encoder. While the vehicle runs along trajectory, the two encoders will measure the incremental distance and the steering angle of the steering wheel respectively. The estimation pose of the vehicle can be calculated from the data of the two encoders by using the dead reckoning method. The vehicle's pose at time step $t_{k}$ is defined by $X_{k}=\left[x_{k}, y_{k}, \theta_{k}\right]^{T}$. The coordinates $\left(x_{k}, y_{k}\right)$ specifies the vehicle's position and the angle $\theta_{k}$ defines the vehicle's orientation in the Cartesian coordinate system. The global coordinate frame $(X, Y)$, shown in Fig.1, is stationary and the origin point $O$ is fixed at the initial 
position of the vehicle. The vehicle local coordinate system is attached to the central point $M$ of the rear axle and its $X$ axis aligns with the rear axis of the vehicle. Initially, the local coordinate frame coincides with the global coordinate frame.

It is assumed that the intelligent vehicle moves along a circular trajectory from time $t_{k}$ to $t_{k+1}$. Its position $\left(x_{k+1}, y_{k+1}\right)$ and orientation $\theta_{k+1}$ at time $t_{k+1}$ is given by:

$$
\left\{\begin{array}{l}
x_{k+1}=x_{k}+\Delta S_{k} \cos \left(\theta_{k}+\Delta \theta_{k} / 2\right) \\
y_{k+1}=y_{k}+\Delta S_{k} \sin \left(\theta_{k}+\Delta \theta_{k} / 2\right) \\
\theta_{k+1}=\theta_{k}+\Delta \theta_{k}
\end{array}\right.
$$

where $\Delta S_{k}$ is the incremental distance and $\Delta \theta_{k}$ is the elementary rotation from state $k$ to $k+1$.

The value of the incremental distance $\Delta S_{k}$ can be got from the driving encoder and the steering angle $\psi_{k}$ of the virtual front wheel at time $t_{k}$ can be calculated from the steering encoder. From Fig.1, the relationship between the steering angle $\psi_{k}$ of the virtual front wheel and the elementary rotation $\Delta \theta_{k}$ of the vehicle can be established as followed.

$$
\Delta \theta_{k}=\frac{\Delta S_{k} \tan \left(\psi_{k}\right)}{L}
$$

where $L$ is the length from the rear axle to the front axle.

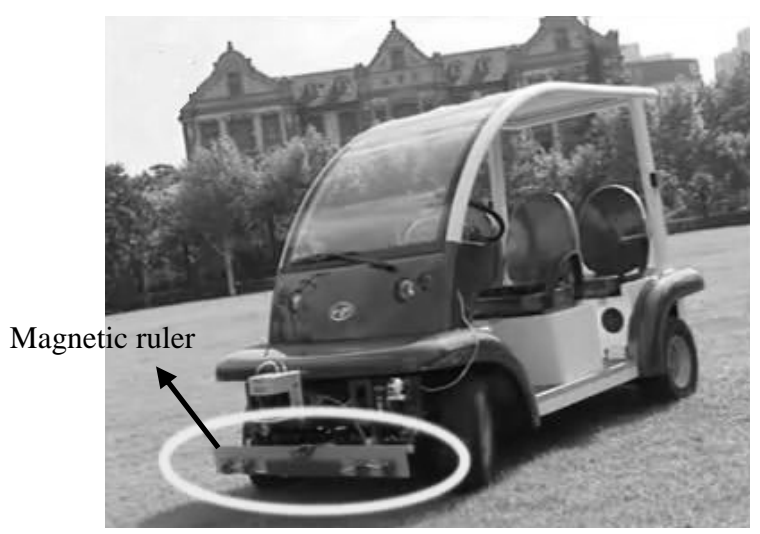

Fig. 2. Intelligent Vehicle with the Magnetic Ruler

\subsection{Magnetic Sensing System}

Magnetic sensing technology has been proven to one promising way for providing absolute positioning reference for intelligent vehicle localization. In the magnetic sensing system, there are two main subsystem , the magnetic marker and the magnetic ruler. The magnetic marker is made of $\mathrm{NdFeB}$ material in a cylindrical shape with a diameter of $2.5 \mathrm{~cm}$ and a length of $2.5 \mathrm{~cm}$, which is embedded under the road surface along the predefined trajectory to provide the magnetic field signal. The magnetic ruler consists of thirteen Anisotropic Magnetoresistive (AMR) sensors with the length of 1 meter. The ruler is attached on the front bumper of the intelligent vehicle with a height of $18 \mathrm{~cm}$ from the ground to the AMR sensor, shown in Fig.2. The magnetic ruler can measure the magnetic field of the magnetic marker as low as $85 \mu$ Gauss. It is more sensitive relative to the sensing system based on Halleffect sensor. The lateral offset of the vehicle to the defined trajectory can be calculated from the measurement results of the magnetic ruler by using the peak mapping algorithm and the measurement accuracy of $1 \mathrm{~cm}$ can be obtained. The price of the sensor in this sensing system is approximate $6 \$$, and is low cost relative to the magnetic sensing system based on fluxgate magnetometer.

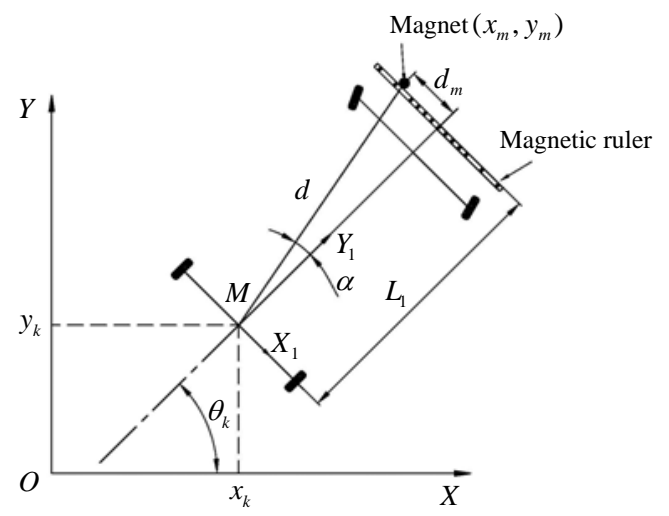

Fig. 3. Measurement Model

When the vehicle is autonomously driven along the trajectory, the magnetic ruler will detect the magnetic markers defined in the global coordinate system and the relative position relationship between the vehicle and the magnetic marker can be established. The rangebearing pair associated to a detected marker, is defined by $(d, \alpha)$ in the local vehicle coordinate system, shown in Fig.3. Therefore, the following equations can be got:

$$
\left\{\begin{array}{l}
d=\sqrt{\left(y_{m}-y_{k}\right)^{2}+\left(x_{m}-x_{k}\right)^{2}} \\
\alpha=\arctan \frac{y_{m}-y_{k}}{x_{m}-x_{k}}-\theta_{k}
\end{array}\right.
$$


where $\left(x_{m}, y_{m}\right)$ is the coordinates of the marker in the global coordinate system.

According to (3), the nonlinear measurement model can be defined as following:

$$
Z_{k}=h\left(X_{k}\right)+v_{k}
$$

where $h\left(X_{k}\right)$ is the nonlinear vector function

$$
h\left(X_{k}\right)=\left[\begin{array}{l}
\sqrt{\left(y_{m}-y_{k}\right)^{2}+\left(x_{m}-x_{k}\right)^{2}} \\
\arctan \frac{y_{m}-y_{k}}{x_{m}-x_{k}}-\theta_{k}
\end{array}\right]
$$

and $v_{k}$ is the Gaussian sensor noise vector.

The range-bearing pair $(d, \alpha)$ is the observation value and $Z_{k}=[d, \alpha]^{T}$ is calculated from the measurement results of the magnetic ruler as follows:

$$
Z_{k}=\left[\begin{array}{l}
d \\
\alpha
\end{array}\right]=\left[\begin{array}{l}
\sqrt{d_{m}^{2}+L_{1}^{2}} \\
\arctan \frac{d_{m}}{L_{1}}
\end{array}\right]
$$

where $d_{m}$ is the distance from the central point of the known marker position $\left(x_{m}, y_{m}\right)$ to the central point $M$ of the magnetic ruler. $L_{1}$ is the distance from the central point of the magnetic ruler to the central point $M$ of the rear axle.

\section{Data Fusion of Odometry and Magnetic Ruler}

When the magnetic ruler detects the magnetic marker, the absolute positioning reference will be provided. A correction of the odometric estimation is performed by using an UKF algorithm and good results usually can be obtained. However, several external disturbance sources, such as earth magnetic filed and AC-generated magnetic field, will produce some noises. There are also some occasions when a single magnetic marker is missed or a fault marker is detected. In these cases, the large error will occur in position estimation. It is necessary to utilize the data association method to judge the validity of the observations during the state update phase in the UKF.

\subsection{System Architecture}

Fig.4 shows the architecture of the intelligent vehicle localization system. The prior position estimation of the vehicle can be calculated from odometry. The magnetic ruler can measure the lateral offset of the vehicle and provide the observations. The estimations from the odometry and the observations are compared by using the data association method. If the innovation distance $\tau_{k}=v_{k}^{T} S_{k}^{-1} v_{k}$ is more than the validation gating $\sigma$, the observations should be rejected and keep the estimation $\hat{X}_{k \mid k-1}$.

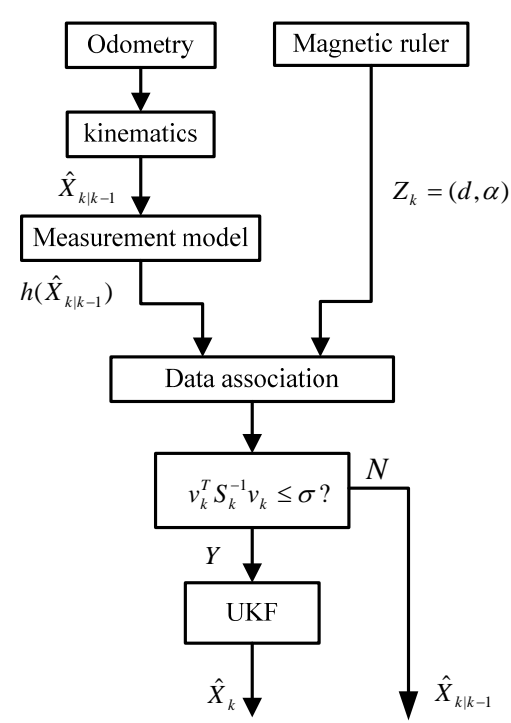

Fig. 4. Block Diagram of the Localization System

\subsection{Unscented Kalman Filter}

The UKF is a recursive MMSE estimator that addresses some of the approximation issues of the EKF. In the UKF, the state distribution is represented by a Gaussian random variable (GRV), but it is specified using a minimal set of deterministically chosen sample points. These sample points completely capture the true mean and covariance of the GRV only with the $3^{\text {rd }}$ or higher order errors ${ }^{16}$.

The system model of the intelligent vehicle defined by the kinematic nonlinear difference equation with a state vector $X_{k}=\left[x_{k}, y_{k}, \theta_{k}\right]^{T}$ and an input vector $u_{k}=\left[\Delta S_{k}, \Delta \theta_{k}\right]^{T}$ can be written in the compact form:

$$
X_{k}=f\left(X_{k-1}, u_{k}, w_{k-1}\right)
$$

where the random variable $w_{k-1}$ with covariance matrix $P_{w}$ represents the process noise.

The nonlinear measurement model can be defined as:

$$
Z_{k}=h\left(X_{k}, v_{k}\right)
$$


where the random variable $v_{k}$ with covariance matrix $P_{v}$ represents the measurement noise. The nonlinear function $h\left(\right.$.) relates the state $X_{k}$ to the measurement $Z_{k}$. The process of the UKF is as follows:

1) If the mean of the $n$-dimensional random variable $X_{k}$ is $\hat{X}_{k \mid k}$ and the covariance of $X_{k}$ is $P_{k k k}$, $X_{k}$ can be approximated by $2 n+1$ weighted samples or sigma points selected by the following algorithm:

$$
\begin{aligned}
& \left\{\begin{array}{l}
\chi_{0, k \mid k}=\hat{X}_{k \mid k} \\
\chi_{i, k \mid k}=\hat{X}_{k \mid k}+\left(\sqrt{(n+\kappa) P_{k \mid k}}\right)_{i} \\
\chi_{n+i, k \mid k}=\hat{X}_{k \mid k}-\left(\sqrt{(n+\kappa) P_{k \mid k}}\right)_{i}
\end{array}\right. \\
& \left\{\begin{array}{l}
W_{0}=\kappa /(n+\kappa) \\
W_{i}=1 /\{2(n+\kappa)\}
\end{array}, i=1,2, \ldots, n\right.
\end{aligned}
$$

where $\kappa \in R,\left(\sqrt{(n+\kappa) P_{k / k}}\right)_{i}$ is the $i$-th column of the square root of matrix $(n+\kappa) P_{k \mid k}$, and $W_{i}$ is the weight that is associated with the $i$-th point.

2) Given the set of samples generated by (9), each sigma point is instantiated through the process model(7) to yield a set of transformed samples

$$
\chi_{i, k k-1}=f\left(\chi_{i, k-1}, u_{k-1}, k-1\right)
$$

3) The predicted mean is computed as

$$
\hat{X}_{k \mid k-1}=\sum_{i=0}^{2 n} W_{i} \chi_{i, k k-1}
$$

4) The predicted covariance is computed as

$$
P_{k \mid k-1}=\sum_{i=0}^{2 n} W_{i}\left(\chi_{i, k \mid k-1}-\hat{X}_{k \mid k-1}\right)\left(\chi_{i, k \mid k-1}-\hat{X}_{k \mid k-1}\right)^{T}+P_{w}
$$

5) The predicted observation is determined by

$$
\hat{Z}_{k \mid k-1}=\sum_{i=0}^{2 n} W_{i} \gamma_{i, k \mid k-1}
$$

where $\gamma_{i, k k-1}$ are instantiations of projected sigma points for each variable and calculated by $\gamma_{i, k \mid k-1}=h\left(\chi_{i, k \mid k-1}, k-1\right)$.

6) The results calculated by (14) are used to estimated the vehicle's pose as

$$
\hat{X}_{k}=\hat{X}_{k \mid k-1}+K_{k}\left(Z_{k}-\hat{Z}_{k \mid k-1}\right)
$$

where $K_{k}=P_{x_{k} y_{k}} P_{y_{k} y_{k}}^{-1}$. The covariance is determined by

$$
P_{y_{k} y_{k}}=\sum_{i=0}^{2 n} W_{i}\left(\gamma_{i, k \mid k-1}-\hat{Z}_{k \mid k-1}\right)\left(\gamma_{i, k \mid k-1}-\hat{Z}_{k \mid k-1}\right)^{T}+P_{v}
$$

and the cross correlation matrix is determined by

$$
P_{x_{k} y_{k}}=\sum_{i=0}^{2 n} W_{i}\left(\chi_{i, k \mid k-1}-\hat{X}_{k \mid k-1}\right)\left(\gamma_{i, k \mid k-1}-\hat{Z}_{k \mid k-1}\right)^{T}
$$

7) Finally the updated covariance is determined by

$$
P_{k}=P_{k \mid k-1}-K_{k} P_{y k y_{k}} K_{k}^{T}
$$

\subsection{Data Association}

Data association (DA) derives the correlation between a measurement and a landmark. It is crucial for the operation of any estimation process ${ }^{17}$. In this paper, the data association based on the nearest neighboour method is utilized to evaluate the consistency between the prediction range-bearing pair from the odometry and the measurement range-bearing pair from the magnetic sensing system.

The nearest neighbour is a classical approach that determines the difference between the prediction results and the measurement results. When the magnetic ruler outputs a measurement result, only the result, which is close enough to the prediction result, is considered to be the possible candidate. The criterion of validation gate $\sigma$ is given by:

$$
\tau_{k}=v_{k}^{T} S_{k}^{-1} v_{k} \leq \sigma, i=1,2, \ldots, N ; j=1,2, \ldots, M
$$

where $v_{k}=Z_{k}-\hat{Z}_{k \mid k-1}$, and $S_{k}$ is the covariance of the innovation $v_{k}, \tau_{k}$ is the Mahalanobis distance ${ }^{18}$.

Note that since $v_{k}$ is a Gaussian random variable, Mahalanobis distance $\tau_{k}$ is a random variable following the $\chi^{2}$ distribution. Thus, the validation gate $\sigma$ is used to decide whether the measurement result $Z_{k}$ is a close enough match to the predicted result. From the $\chi^{2}$ distribution (Tab.1), we know $\tau_{k} \leq 6.635$ with a probability of 0.99 . Here we set $\sigma=6.635$.

In order to assure the confidence level of the observations from the magnetic ruler, before the vehicle pose is updated using the observations, the validity of 
the measurement range-bearing pair should be validated by adopting the data association. If the Mahalanobis distance $\tau_{k}$ is more than the validation gate, the measurement range-bearing pair from the magnetic sensing system should be rejected.

Tab. $1 \chi^{2}$ Distribution

\begin{tabular}{|c|c|c|c|c|}
\hline $90 \%$ & $95 \%$ & $97.5 \%$ & $99 \%$ & $99.5 \%$ \\
\hline 2.706 & 3.841 & 5.024 & 6.635 & 7.879 \\
\hline
\end{tabular}

\section{Experimental Results}

In order to analyze the fusion performance of the proposed method based on UKF, the field experiments have been performed. The magnetic ruler is installed on the front bumper of the vehicle and the magnetic markers are embedded under the road surface along the defined trajectory. The magnetic ruler can measure the lateral offset of the vehicle with less than $1 \mathrm{~cm}$ error. There are altogether twenty magnetic markers along the defined trajectory with the spacing of $2 \mathrm{~m}$.

The experimental results indicate the implementation of the UKF method presented in Section 3.2. The initial true state of the system is $X(0)=(0,0, p i / 2)$, and the initial covariance matrix of the initial state is $P_{0 \mid 0}=\left[1 \times 10^{-2}, 0,0 ; 0,1 \times 10^{-2}, 0 ; 0,0,1 \times 10^{-2}\right]$. Owing to $n=3$, $\kappa$ is chosen to be zero in accordance with $n+\kappa=3$. Process noise is Gaussian white noise, and the process noise covariance matrix is $P_{w}=\left[1 \times 10^{-4}, 0,0 ; 0,1 \times 10^{-4}, 0 ; 0,0,8.73 \times 10^{-3}\right]$. Measurement noise is Gaussian white noise, and the measurement noise covariance matrix of the magnetic ruler is $P_{v}=\left[1 \times 10^{-4}, 0 ; 0,3.1 \times 10^{-4}\right]$.

Fig.5 shows the localization results without fault makers based on the UKF algorithm. Owing to the accumulative error in dead reckoning process, the localization results from the dead reckoning are not satisfactory for the precise vehicle localization. With the fusion of the odometry and the magnetic ruler by using the UKF algorithm, the localization errors have been reduced obviously. However, while the magnetic sensing system suffers from some large disturbances or the magnetic ruler detects the fault markers, the large error will occur in position estimation. As shown in Fig. 6 , there are two fault markers along the trajectory, the localization results are obviously destroyed due to the two fault markers.

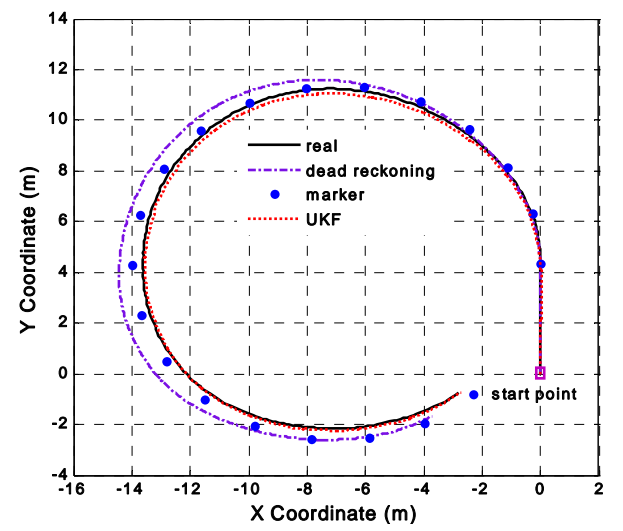

Fig. 5. Localization Results without Fault Marker

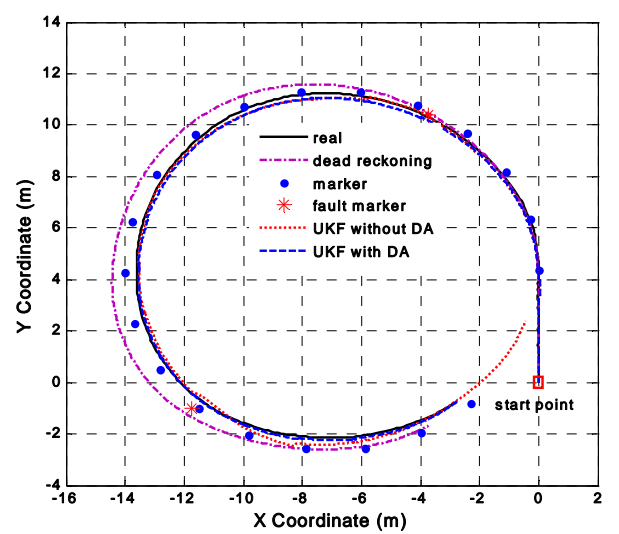

Fig. 6. Localization Results with Two Fault Markers

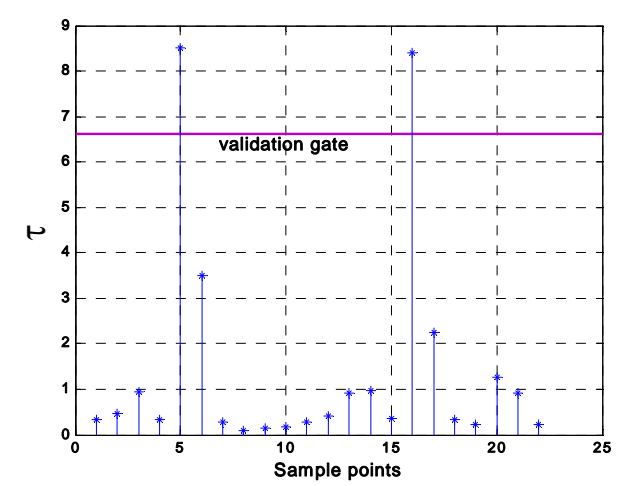

Fig. 7. Innovation Distance

In order to realize the robust intelligent vehicle localization, the data association method is proposed to validate the observations from the magnetic ruler in the UKF update stage. When the innovation distance between the observation and the estimation is more than the validation gate, the observation should be rejected. 
From Fig.7, there are two innovation distance values above the validation gate owing to the two fault markers.

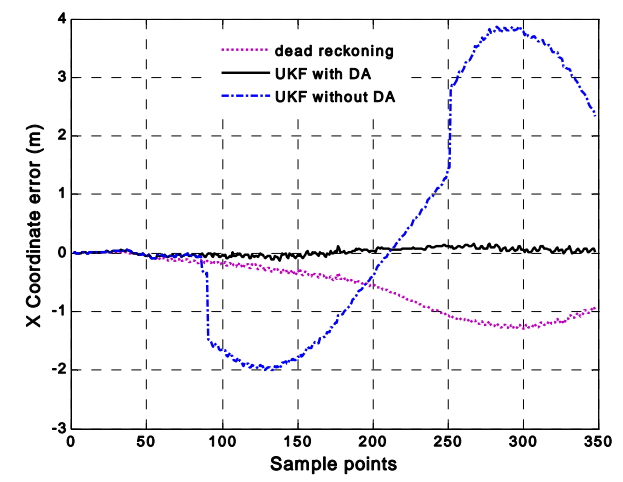

Fig. 8. Localization X Coordinate Error

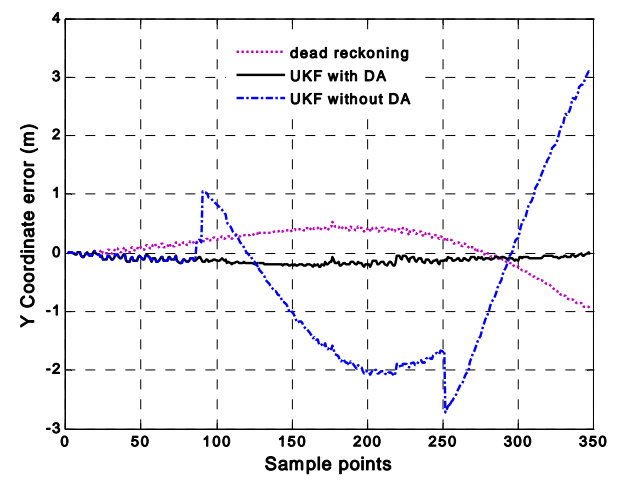

Fig. 9. Localization Y Coordinate Error

Fig.8 and Fig.9 show the large localization errors by utilizing the UKF without data association method. There are two steep position change. When the magnetic ruler detects the fault marker, which is not belong to the position reference marker, the large steep position errors are produced due to the incorrect position mesurement reference from the fault marker. The UKF with data association method can validate the observations from the magnetic ruler and filter the mesurement reference from the fault marker. The experiment results in Fig.8 and Fig.9 show that the good localization results are obtained by utilizing the UKF with data association method.

\section{Conclusions}

The magnetic ruler in the magnetic sensing system can detect the magnetic marker embedded under the road surface and provide the absolute positioning reference for the mobile robot localization under all kinds of weather conditions or environmental conditions. The accumulative error in dead reckoning process can be obviously reduced by fusing the measurement results from the magnetic ruler. The performance of the robot localization system is improved by adopting the data association method in the UKF algorithm to judge the validity of the observations from the magnetic ruler.

\section{Acknowledgements}

The work was supported by the Shanghai Science and Technology Action Program for World Expo (10dz0581100).

\section{References}

1. P. Bonnifait, P. Bouron, P. Crubille, and D. Meizel, Data fusion of four ABS sensors and GPS for an enhanced localization of car-like vehicles, Proceedings of the 2001 IEEE International Conference on Robotics \& Automation (Seoul, Korea, 2001), pp. 21-26.

2. J. Huang, H. S. Tan, A low-order DGPS-based vehicle positioning system under urban environment, IEEE/ASME Transactions on Mechatronics. 11(5) (2006) 567-575.

3. T. Aono, K. Fujii, S. Hatsumoto, and T. Kamiya, Positioning of vehicle on undulating ground using GPS and dead reckoning, Proceedings of the 1998 IEEE International Conference on Robotics \& Automation (Leuven, Belgium, 1998), pp. 3443-3448.

4. M. Chen, B. Jiang, J. Zou, X. Feng, Robust adaptive tracking control of the underwater robot with input nonlinearity using neural networks, International Journal of Computational Intelligence Systems. 3(5)(2010)646655.

5. C. C. Tsai, A localization system of a mobile robot by fusing dead reckoning and ultrasonic measurements, IEEE Transactions on Instrumentation and Measurement. 47(5) (1998) 1399-1404.

6. S. Lee, J. B. Song, Robust mobile robot localization using optical flow sensors and encoders, Proceedings of the 2004 IEEE International Conference on Robotics \& Automation (New Orleans, 2004), pp.1039-1044.

7. T. S. Jin, J. M. Lee, and S. K. Tso, A new approach using sensor data fusion for mobile robot navigation, Robotica. 22(1) (2004) 51-59.

8. C. Y. Chan, Magnetic sensing as a position reference system for ground vehicle control, IEEE Transactions on Instrumentation and measurement. 51(1) (2002) 43-52.

9. S. M. Donecker, T. A. Lasky, and B. Ravani, A mechatronic sensing system for vehicle guidance and control, IEEE/ASME Transactions on mechatronics. 8(4) (2003) 500-510.

10. H. S. Tan, J. Guldner, S. Patwardhan, C. Chen, and B. Bougler, Development of an automated steering vehicle based on roadway magnets-A case study of mechatronic 
system design, IEEE/ASME Trans. Mechatronics. 4(3) (1999) 258-272.

11. L.C. Bento, U. Nunes, F. Moita, and A. Surrecio, Sensor fusion for precise autonomous vehicle navigation in outdoor semi-structured environments, In Proc. of IEEE Int. Conf. on Intelligent Transportation Systems (Vienna, Austria, 2005), pp.245-250.

12. H. Akashi, H. Kumamoto, Random sampling approach to state estimation in switching environments, Automatica, 13 (1977) 429-434.

13. E. A. Wan, R. V. de Merwe, The unscented kalman filter for nonlinear estimation, Proceedings of Symposium 2000 on Adaptive Systems for Signal Processing, Communication and Control,AS-SPCC (Lake Louise, Alberta, Canada,2000), pp.153-158.

14. H. G. Xu, C. X. Wang, M. Yang, and R. Q. Yang, Position estimation for intelligent vehicles using an unscented Kalman filter, International Journal of Vehicle Autonomous Systems. 6(1/2) (2008) 186-194.

15. W. H. Wang, F. G. Huo, H. C. Tan, H. Bubb, A framework for function allocation in intelligent driver interface design for comfort and safety. International Journal of Computational Intelligence Systems. 3(5)(2010)531-541.

16. R. V. de Merwe, A. Doucet, N. de Freitas, and E. Wan, The unscented particle filter, Technical Report CUED/FINFENG/TR 380 (Cambridge University Engineering Department, 2000).

17. N. M. Kwok, A. B. Rad, A modified particle filter for simultaneous localization and mapping. Journal of intelligent and robotics systems. 46(4) (2006) 365-382.

18. S. Zhang, L. H. Xie, and M. Adams, An efficient data association approach to simultaneous localization and map building, Proceedings of the 2004 IEEE International Conference on Robotics \& Automation (New Orleans, LA, United states, 2004), pp. 854-859. 